\title{
Advances in Electrical Source Imaging: A Review of the Current Approaches, Applications and Challenges
}

\author{
Ioannis Zorzos ${ }^{1} \mathbb{D}$, Ioannis Kakkos ${ }^{1, *}$, Errikos M. Ventouras ${ }^{2}$ and George K. Matsopoulos ${ }^{1}$ \\ 1 Laboratory of Biomedical Optics \& Applied Biophysics, School of Electrical and Computer Engineering, \\ National Technical University of Athens, 9, Iroon Polytechniou Street, Zografos, 15780 Athens, Greece; \\ zorzos_i@biomig.ntua.gr (I.Z.); gmatso@esd.ece.ntua.gr (G.K.M.) \\ 2 Department of Biomedical Engineering, University of West Attica, 17, Ag. Spyridonos Street, Egaleo, \\ 12243 Athens, Greece; ericvent@uniwa.gr \\ * Correspondence: ioakakkos@gmail.com; Tel.: +30-2107723577
}

check for updates

Citation: Zorzos, I.; Kakkos, I.; Ventouras, E.M.; Matsopoulos, G.K. Advances in Electrical Source Imaging: A Review of the Current Approaches, Applications and Challenges. Signals 2021, 2, 378-391. https: / / doi.org/10.3390/ signals2030024

Academic Editor: Giorgos Giannakakis

Received: 27 February 2021

Accepted: 21 June 2021

Published: 24 June 2021

Publisher's Note: MDPI stays neutral with regard to jurisdictional claims in published maps and institutional affiliations.

Copyright: (c) 2021 by the authors. Licensee MDPI, Basel, Switzerland. This article is an open access article distributed under the terms and conditions of the Creative Commons Attribution (CC BY) license (https:// creativecommons.org/licenses/by/ $4.0 /)$.

\begin{abstract}
Brain source localization has been consistently implemented over the recent years to elucidate complex brain operations, pairing the high temporal resolution of the EEG with the high spatial estimation of the estimated sources. This review paper aims to present the basic principles of Electrical source imaging (ESI) in the context of the recent progress for solving the forward and the inverse problems, and highlight the advantages and limitations of the different approaches. As such, a synthesis of the current state-of-the-art methodological aspects is provided, offering a complete overview of the present advances with regard to the ESI solutions. Moreover, the new dimensions for the analysis of the brain processes are indicated in terms of clinical and cognitive ESI applications, while the prevailing challenges and limitations are thoroughly discussed, providing insights for future approaches that could help to alleviate methodological and technical shortcomings.
\end{abstract}

Keywords: EEG; source imaging; source localization; applications; challenges

\section{Introduction}

Electrical Source Imaging (ESI) is an imaging technique utilized to localize the activated brain regions by incorporating temporal and spatial components from electroencephalogram (EEG) signals. Although this imaging method provides fine temporal resolution compared to other imaging methods, such as the functional magnetic resonance imaging (fMRI), the number of electrodes used to record the brain electrical potentials is significantly smaller than the number of activated neurons, rendering EEG source localization an ill-posed problem. To determine the generating source of the brain's electrical activity, the scalp potentials (i.e., the potentials created by the synchronous activation of pyramidal neurons in the brain [1], which are propagated through the different tissues that compose the head) are recorded by an array of surface electrodes. Then, an estimation of the signals origin is calculated through Poisson's equation [2]. Poisson's equation is directly derived from Maxwell's equations, given that the head tissues have the permeability of free space, while the localization of the activated regions within the brain is commonly referred to in the literature as the inverse problem. On the contrary, computing the scalp potentials from a given source (a prerequisite to calculate inverse solutions) is designated as the EEG forward problem. As such, given that Poisson's equation highly depends on the accurate values of the electrical and geometrical properties (thickness, conductivity, etc.) of the head tissues (scalp, skull, cerebrospinal fluid (CSF), brain cavities, etc.), it is evident that a precise head model is critical to solve the forward problem correctly. To address this, anatomical information can be provided by magnetic resonance imaging (MRI). Nevertheless, it is extremely difficult to obtain head MRI in a subject-specific fashion. Thus, a large number of studies rely on template anatomical information [3-5]. Having a proper head model also facilitates the correct electrode positioning, since the location of each electrode varies for 
each individual (although to a small degree). In this context, the anatomical information as well as the corresponding electrode locations comprise the Leadfield matrix, the accurate calculation of which is closely related to the accuracy of the solutions of the inverse problem [6]. The EEG source localization has puzzled researchers since a vast variety of combinations of sources can produce the same signals recorded by the EEG [7]. This is a fundamental problem of EEG source localization, because even with a flawless head model and an excellent noiseless signal, the number of equations that can be solved is minuscule compared to the number of dipoles (source points) within the brain, leading to non-unique possible solutions. This non-uniqueness can be handled with mathematical, anatomical or neurophysical constraints about the location and the direction of dipoles [8,9]. Therefore, even if non-uniqueness is not guaranteed, these a priori assumptions are utilized, in order to reduce the computational needs for the source estimation and increase the accuracy of the solutions.

However, owing to recent advances which incorporate novel methodologies as well as the introduction of machine learning approaches in solving the inverse problem [10,11], the required time and the computational resources for the solution have been significantly reduced. Furthermore, sophisticated algorithms have diminished the localization error efficiently, estimating the location and activation of the different cortical regions [12]. This is evident in several applications, ranging from detecting and assisting the therapy of epilepsy, to interpreting emotions from facial expressions $[6,13]$.

This continually updated progress in both the forward and the inverse problem solutions, designates ESI as a "living" tool, revolutionizing neurodynamic analysis in clinical, biomedical and cognitive applications. On this premise, the presentation of the current advancements in EEG source localization have been the key motivation in this review paper, which aims to highlight the different approaches of the multiple state-of-theart ESI algorithms, pinpointing the advantages and disadvantages of each method, as well as their related limitations that affect the spatial resolution or the computational complexity. Moreover, the recent clinical and cognitive applications of EEG source localization are discussed, as well as the implications and challenges occurring due to methodological and technical constraints, proposing future actions.

\section{State-of-the-Art}

In this paper, current ESI implementation approaches, trends and challenges are presented. The databases examined for the synthesis of the studies presented werePubMed, Scopus, ScienceDirect and Google Scholar. Eligibility criteria included research works that incorporate recent advancements (published after 2015) regarding forward and inverse solutions, clinical and cognitive applications and methodological/algorithmic aspects of source imaging.

\subsection{Forward Problem}

To solve the EEG forward problem, three main configurations are required-(a) the head and source models (i.e., the location of the solution points in the brain), (b) the electrode alignment on the head model, and (c) the Leadfield matrix using the channel locations in relation to the anatomical information of the head model. As such, the solution's accuracy highly depends on the efficient generation and composition of the above. The basic components and the flow of processes are shown in Figure 1.

Regarding the creation of the head model, the integration of anatomical data has rendered earlier widely adopted spherical head models outdated [14]. Converting MRI images into head models is a time-consuming process with a high computational cost, and this procedure presents optimal results in terms of localization accuracy. Nevertheless, subject-specific MRI recordings are not always available, requiring the head model to be constructed through computational methods. In this regard, however, it is important to emphasize that the sophistication of the head models does not display a linear trend with ESI localization, leading to high implementation complexity (e.g., inclusion of skull 
spongiosa) to demand significant computational resources, with little to no significant impact in spatial accuracy $[15,16]$.

The most common methods utilized for head-modeling are the boundary element methods (BEMs), finite difference methods (FDMs) and the finite element methods (FEMs) [17-20]. The Projective Method (PM) (that incorporates the mathematical descriptors of surfaces, such as Fourier descriptors, and dimensionality reduction methods, such as Principal Component Analysis), is also well established, albeit less common [21,22]. Of note is that for PM, in contrast with the aforementioned methods, the time required to extrude the head model and the computational resources is drastically reduced, but with lower spatial resolution [23].

Regarding the frequently employed algorithms, FEM and FDM provide more analytical outcomes and can tackle inhomogeneity problems, which is vital if the head model requires the modeling of anisotropic properties of white matter and the skull. On the other hand, BEM presents lower computational time and accuracy in comparison to the FEM and FDM, being unable to treat inhomogeneous and nonlinear problems.

Even though the FEM approach provides a more well-established detailed model, since it incorporates more than the three standard head tissues, its universal implementation has been hamstrung due to its computational needs and lack of open-source tools incorporating a complete FEM approach. However, the Fieldtrip-SimBio pipeline has been recently introduced, providing an integrated EEG forward problem solution, while employing an FEM-created head model [24]. On this premise, it should also be noted that subject-specific MRIs are not always possible to be recorded. For this reason, an averaged (standardized) volume conductor model using the ICBM 152 anatomical template and the FEM ("The New York Head") was created, showing promising results in ESI, compared to other standardized FEM and BEM approaches [25]. While FEM effectiveness is shown to be comparable with analytical solutions [26], adverse issues could occur due to skull leakage effects (i.e., the inhomogeneity of skull thickness), which could lead the implemented model to present similar properties to a simple three-layer sphere model [27]. In order to overcome this inconsistency, different FEM approaches are used, deviating from the standard continuous Galerkin-FEM method (CG-FEM) by creating a mixed or discontinuous Galerkin-FEM utilizing the subtraction approach [28,29]. Evidence suggests that this solution combines the benefits from both approaches, decreasing the skull leakage effect [19]. Furthermore, using analytical expressions combined with the subtraction approach, the accuracy of the forward problem solution was increased compared to other numerical approaches with similar computational costs [30]. Increments in the precision of the EEG forward problem results have also been demonstrated by the incorporation of tissue inhomogeneity, even within the gray and white matter [31]. Apart from FEM, computationally efficient and accurate solutions have also been achieved with the introduction of anisotropic conductivity equations and utilization of the reciprocity theorem [32] on FDM head models (AFDRM-NZ) [33]. In this regard, anisotropic conductivity values are integrated by employing a set of surface integral equations aligning the produced solutions with the analytical results [34]. Of note is that the skull conductivity and, therefore, the correct modeling is a pivotal point, which, due to head abnormalities or insufficient imaging tools, can be challenging to approximate [35]. To address this issue, Bayesian Approximation Error (BAE) approaches displayed high efficiency in reducing the source localization error by several millimeters, enhancing spatial accuracy, which is crucial in clinical applications [36]. 

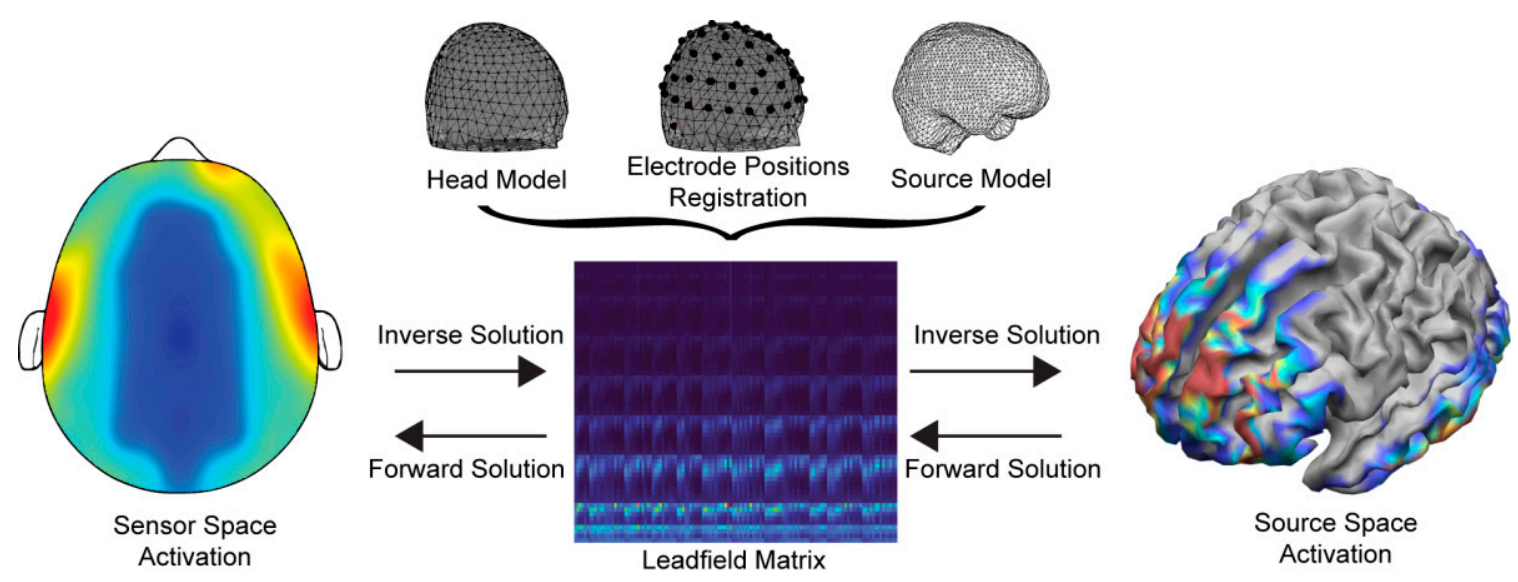

Figure 1. Information flow and basic components of the forward and inverse problems.

\subsection{Inverse Problem}

Contrary to the forward problem, the inverse problem cannot be uniquely solved if there are no a priori restrictions about the source locations [37]. This fact has led researchers to incorporate various mathematical constraints preceding the source estimation, thus reducing the number of possible solutions deriving from the recorded data. Such constraints are the core of many of the conventional methods used to solve the inverse problem. The inverse methods that are commonly used fall into two main categories-non-parametric (non- adaptive) and parametric (adaptive) distributed source imaging.

Although there is an extremely large number of ESI methodologies for parametric (e.g., Beamformers, Multiple Signal Classification) and non-parametric (e.g., Minimum Norm Estimate, Focal Underdetermined System Solution, Local Auto-Regressive Average) distributed source imaging methods of the inverse solution, in this paper, we focus on the most commonly employed algorithms, to highlight the progress of ESI. For an extensive review of the mathematical aspects and properties of several of these conventional methods and their variants, that are beyond the scope of this review paper, please refer to the thorough reviews [38,39].

The most frequent non-parametric algorithms employed are the minimum norm estimate (MNE) solution [40] and its depth-weighted variant (dw-MNE) [41,42], although several other designs take into account the same principles with modified settings and additional parameter incorporation. For instance, the low resolution electromagnetic tomography activity (LORETA) estimates the current density given by the minimum norm solution but with a more sophisticated regularization, utilizing a discrete Laplace operator that selects preferentially spread source ("smooth") distributions, in contrast with the MNE's identity matrix $[43,44]$. On the other hand, parametric distributed source imaging methods commonly include Linear Constrained Minimum Variance (LCMV) beamformers that depend on structurally related filters to provide efficient source localization irrespective of noise covariance [45]. Contrary to the non-parametric approaches for solving the inverse solution, LCMV beamformers isolate the signals produced by different parts of the brain using spatial filters, thus allowing the solution computations to occur independently for each solution point.

Even though conventional methods have been proven to be efficient in determining the activated brain regions, emerging technologies in the fields of Machine and Deep Learning have been recently introduced in ESI. As such, a novel method proposed for solving the inverse problem utilizes the deep recurrent neural network architecture of longshort term memory (LSTM) units in an auto-encoder framework, presenting exceptional mean localization error of less than $5 \mathrm{~mm}$ on single-source simulated data [10]. This architecture is able to model the spatio-temporal information provided by training the network to perceive the correlation between the location of the source and the EEG signals without needing a priori constrictions, normally provided manually by more conventional 
methods. In a similar manner, ConvDip, a convolutional neural network (CNN), has demonstrated a lower normalized mean squared error in ESI solutions compared to that of exact LORETA (eLORETA) and beamformers for a single source, utilizing a shallow CNN with one convolutional layer and two fully connected layers [11]. Compared to the LSTM approach, ConvDip was trained with single time-instances on simulated data but with multiple sources. This is important, since a large variety of inverse solutions, such as eLORETA and LCMV beamformer, rely on noise covariance matrices that are computed with the temporal information of EEG signals, significantly affecting the accuracy of the model if the noise is increased. Moreover, the fact that simple networks can learn the patterns of single time points and predict reasonable inverse solutions is a major point in lowering complexity and consequently computational cost. Additional ESI neural networks frameworks include a denoising AutoEncoder (DST-DAE) consisting of six layers, three encoding blocks and three decoding blocks. This method was able to directly map the EEG and magnetoencephalogram (MEG) signals to the cortical sources, reducing the localization error to less than a millimeter [46]. In this regard, both temporal and spatial information is utilized on synthetic data, in order to extract inverse mapping. The main advantage of this method lies in its resistance and robustness against low Signal-to-Noise ratio (SNR), resulting in efficient source estimation with excellent denoising properties. Added together, the recent advances in Machine Learning ESI estimation indicate the efficacy of the employed procedures in contrast to the traditional model-driven approaches, especially since in all of the data-driven methods, very few or no mathematical priors were used, while the need for optimizing parameters for new data is absent.

Apart from Deep Learning designs, recent studies include hierarchical Bayesian analysis methods for source localization. The importance of Bayesian Models relies on the incorporation of statistical a priori information about the sources, eliminating common problems such as ghost sources and uncorrelated activation transition [39]. The main advantage of such solutions is the combination of data-driven learning with sparse priors, minimizing the cost function and maximizing the probability of correlated sources [47]. Most recently, a modified Bayesian approach was introduced, applying the $\ell_{20}$ mixed norm instead of $\ell_{21}$ (primarily used with Bayesian Methods) and multivariate Bernoulli Laplacian priors [12], with the main difference between the Bayesian Models being the probability distributions for the correlation of the sources. This method was able to provide sparser solutions, minimizing the underestimation of the intensity of activations, indicating higher localization performance than other conventional Bayesian methods, in both simulated and in real auditory and visual evoked data but at high computational cost, requiring almost 58 times more time than the $\ell_{21}$ mixed norm approaches. In a related study [48], a computational efficient Expectation-Maximization algorithm with the use of steady-state Kalman Filter (SS-KF) and steady-state Fixed Interval Smoother (SS-FIS) provided a significant performance enhancement compared to other existing methods, while encapsulating the spatial dependencies between the sources. The computational burden mitigation relies on the use of SS-KF and SS-FIS that are computed once throughout the estimation of the sources without loss of model accuracy, subsequently reducing 12-fold the time needed for results output compared to the full KF/FIS method. It should be pointed out that the aforementioned methods present a theoretically zero localization error, outperforming the solutions provided by neural networks. Nevertheless, the advantage of neural networks lies in the small amount of time needed in order to compute the inverse solution. From this standpoint, the ability of a fast calculation of the source estimates is of utmost importance in the aspiration of developing real-time ESI applications in the near future. A comparison of methods presented, illustrating the current state-of-the-art, is shown in Table 1. 
Table 1. Current trends in state-of the-art methods used in ESI.

\begin{tabular}{|c|c|c|c|}
\hline Method & Authors & Advantages & Disadvantages \\
\hline $\begin{array}{l}\text { Recurrent Neural } \\
\text { Network-Long } \\
\text { Short-Term Memory }\end{array}$ & [10] & $\begin{array}{l}\text { Extremely fast computation of source } \\
\text { estimates, once the training has } \\
\text { completed. Can harness the } \\
\text { spatio-temporal information of EEG, } \\
\text { resulting in more robust solution } \\
\text { regarding noise. Great expandability } \\
\text { and room for improvement. }\end{array}$ & $\begin{array}{l}\text { Trained for single sources. Requires a lot of } \\
\text { time for the training session, even if the } \\
\text { model is simple. Worse accuracy than other } \\
\text { models presented. }\end{array}$ \\
\hline $\begin{array}{l}\text { Convolutional Neural } \\
\text { Netrwork }\end{array}$ & [11] & $\begin{array}{l}\text { Simplicity and expandability. Once } \\
\text { trained, produces results extremely fast. }\end{array}$ & $\begin{array}{l}\text { Trained on single time points and does not } \\
\text { incorporate the temporal information of EEG } \\
\text { creating low noise tolerance. Lower accuracy } \\
\text { on multisource scenarios. }\end{array}$ \\
\hline Denoising AutoEncoder & {$[46]$} & $\begin{array}{l}\text { Very high noise tolerance, producing } \\
\text { accurate results even with low SNR. Do } \\
\text { not require mathematical priors. }\end{array}$ & $\begin{array}{l}\text { Requires a lot of time for training and offline } \\
\text { computation of the Leadfield matrix. } \\
\text { Susceptible to overfitting due to vanishing } \\
\text { gradient for complex scenarios. }\end{array}$ \\
\hline $\begin{array}{l}\text { Bayesian } \\
\text { Method-Bernouli } \\
\text { Laplacian priors }\end{array}$ & [12] & $\begin{array}{l}\text { Near-zero mean localization error. } \\
\text { Great recovery and accuracy of dipole } \\
\text { locations in low SNR. Sparser solutions. } \\
\text { Correct estimation of the amplitude of } \\
\text { source currents. }\end{array}$ & $\begin{array}{l}\text { Very high computational cost. Requires } \\
\text { accurate head model for high accuracy. }\end{array}$ \\
\hline $\begin{array}{l}\text { Bayesian } \\
\text { Method-Kalman Filters }\end{array}$ & [49] & $\begin{array}{l}\text { Near-zero mean localization error. } \\
\text { Lower computational cost than other } \\
\text { spatio-temporal dynamic algorithms, } \\
\text { faster than other KF approaches. }\end{array}$ & $\begin{array}{l}\text { Under-estimation of the amplitude of source } \\
\text { currents. Requires a priori information for } \\
\text { the source covariance matrix. }\end{array}$ \\
\hline
\end{tabular}

\section{ESI-Clinical and Cognitive Research Implementations}

Brain source localization provides a new dimension of the recorded EEG data which can be applied in various conditions, in both clinical and cognitive applications. Of note is that new algorithms continuously improve the time/efficiency and accuracy of the ESI solutions. For this reason, the number of applications to which ESI is applied is constantly increasing. As a result, in this review paper, it was impossible to include all the novel applications of source imaging. Our goal was to attempt to illustrate the advances in the field by presenting the most indicative examples, demonstrating the importance of ESI for clinical and cognitive applications.

\subsection{Clinical Applications}

The most important employment of ESI is in clinical situations. The high temporal resolution provided by EEG, combined with the spatial estimation of the sources given by the ESI, provides non-invasive monitoring of specific brain areas that single modality analysis cannot. As such, ESI is commonly used in locating the epileptic foci, vital for epilepsy treatment and/or for subsequent surgery [50]. In a recent long-term study [51], a synchronously recorded high-density EEG and MEG source localization of ictal and interictal activity provided more accurate results when the analysis was conducted for each modality alone than the multi-modal approach. It is also suggested that less emphasis should be given to mid-phase ictal discharge analysis and include the late-peak phase in source solutions. EEG source localization as a single modality technique is also an effective localization technique for focal epilepsy, minimizing the cost of recording different modalities concurrently, requiring additional expensive equipment [52,53].

Apart from the obvious economic and scientific benefits of ESI applications, the noninvasiveness of source localization holds significant social and healthcare advantages since (multiple) intracranial invasive recordings can be avoided in certain types of epilepsy, thus relieving preoperative anxiety from patients as well as risk of surgical site infections. On 
this account, the prevalent utilization of EEG source localization is in presurgical evaluation of drug-resisting epileptic patients [13]. During this procedure, the information provided by the ESI about epileptiform discharges can have significant impact in the management plan, in terms of placing more accurately the intracranial electrodes $[54,55]$. The identification of the Seizure Onset Zones can not only define the exact cortical structures for surgical extraction but also provide important information concerning the neurodynamic state of the epileptic brain activity, especially in generalized (e.g., absence epilepsy) [56] or focal epilepsies (e.g., structural, non-lesional epilepsy) [52,57,58].

Although high-density EEG is not always available, low-density EEG source localization has been consistently effective in localizing epileptic brain activity, achieving over $90 \%$ accuracy in some cases, while indicating that performance could be further enhanced by including connectivity analysis [58-60]. ESI has also been employed in pediatric epilepsy. In a recent study [61], using a visual working-memory task as a stimulus and a source localization framework, critical biomarkers (that could not be detected by sensor-level analysis alone) were identified, leading to a more localized and specified treatment policy.

The fine temporal communication of the different brain regions in source space has also been applied in neurodegenerative diseases [62]. Frequently, it is used in the evaluation of Alzheimer's disease progress or even in its prognosis, since the brain activation related to cognitive performance is commonly altered in the disease's progression [63]. As such, eLORETA and connectivity analysis has been adopted for Alzheimer's disease diagnosis $[64,65]$. Another interesting approach is the use of ESI in localizing the origin of epileptic spikes using the differences created by non-Rapid Eye Movement (NREM) and Rapid Eye Movement (REM) sleep [66]. In view of the fact that EEG has been consistently employed to study sleep-related cycles and disorders, source localization can efficiently identify the main areas that are involved in the sleep onset transition and the activity alterations in the brain regions affected by sleep deprivation recovery $[67,68]$. Furthermore, ESI is effective in detecting the areas and the associated activity during different NREM sleep stages with the use of MEG and EEG recordings [69], the areas responsible for generating sleep spindles [70] and slow wave generation [71]. Interestingly, source localization has been applied for sleepwalking and sleep terror origin decoding, used not only to estimate the brain areas that were affected by the sleep arousal disorder, but also for computing the decrement of slow wave activity power [72].

Despite the prognostic and diagnostic capabilities of ESI, rehabilitation processes can also be substantially supported. In this context, distinguishing direction by decoding movement intensions on people suffering from motor deficits (such as stroke patients) is of utmost importance in the optimal management and evaluation of rehabilitation planning. Multiple approaches have contributed in classifying multi-direction hand movement, using weighted MNE source localization [73], or Overlapping Averaging weighted MNE (OA-wMNE) [74]. Moreover, detecting the brain source locations of complex imaginary movements, like flexion, extension, supination and pronation, is also feasible with source localization methods and feature selection algorithms [75,76]. On this premise, ESI has been employed to assess the allocation of brain functions with regard to the attempts made by stroke patients in limb movement [77]. Recent approaches have employed highdensity EEG and beamformer techniques to facilitate functional connectivity analysis, thus measuring the connection between alpha and beta band coherence with motor learning and cortical plasticity of post-stroke impairments [78-80].

\subsection{Cognitive Applications}

Apart from the clinical applications, brain source localization helps in gradually elucidating the underlying neural substrates in various conditions and mental states. As such, ESI has been consistently utilized in the decryption of cognitive functions in various tasks, such as word interpretation and fatigue analysis.

Multiple paradigms include source localization in Event-Related Potentials (ERPs) to illuminate the brain activity related to certain stimuli or events. For instance, event- 
related responses in Go-Nogo tasks combined with ESI have been employed to identify and interpret somatosensory response inhibition [81,82]. Specifically, ERP analysis and standardized LORETA (sLORETA) were used to link the differences of amplitude of NogoN2 ERP-component with the changes of activation in the right inferior gyrus, showing that response inhibition is correlated with sensory gain modulation [82]. Another recent study [83] employed beamformers on ERP analysis to provide evidence of the regions responsible for generating the $\mathrm{P} 300$ signal related to attention and memory operations of the brain.

The interpretation of facial expressions and emotions in source space has also been consistently investigated in various studies. As such, taking into account that cognitive face processing is evolving during the early stages of life, the employment of LORETA has been able to provide insight into the underlying process [84]. In a similar way, using an MNE algorithm, ESI was able to further advance the brain state decoding by deciphering the neural operations of facial recognition [85], while the utilization of the LAURA method and harnessing of the "Kuleshov effect" has effectively highlighted the facial expression cognitive functions [86]. Furthermore, combining ESI with a self-organizing feature map (SOMF) resulted in accurate categorization of emotions perceived from physiognomic components, (up to $91 \%$ of classification accuracy) [14]. Similar source localization studies have also been conducted for identifying and analyzing the processing of facial features in autism, distinguishing the regions activated in different cases [87].

More recently, ESI has been applied in visual and auditory evoked data, in an effort to understand the underlying brain connections and the related inhibitory networks $[5,88]$. In fact, since source imaging is able to analyze the paths of brain activity through time, connectivity analysis presents a novel prospective in identifying the transmission of information between cortical areas. In this regard, language understanding and the impact on the brain has been studied using connectivity and sLORETA, revealing individual networks active during lexico-semantic processing of single words [89]. Additionally, pattern recognition frameworks combined with ESI have effectively identified common and distinct functional connections (representing a link between the various brain structures) in different workload levels in two working memory tasks [90]. Moreover, in simulated 2D and 3D flight simulation experiments, eLORETA and graph theory was utilized to assess the dynamic rewiring of functional connections among brain areas as a result of mental workload, establishing the basis for potential real-world applications [91].

\section{ESI Challenges and Limitations}

During the last decade, significant advancements have been made regarding ESI methodologies, both in computational time as well as in the accuracy of solutions for the EEG inverse problem. However, the very nature of the inverse problem, given its underdetermined properties, is a major obstruction. Despite the fact that recent approaches have successfully alleviated several limitations, a number of challenges that need to be addressed are still present.

The greatest drawback of EEG, and by extension source localization calculations, is its sensitivity to signal noise, which can consist of background noise, distortions and movement artifacts. These interferences affect greatly the accuracy of the inverse solution, producing ghost sources, or even displace the predicted brain activity areas completely [92]. In this context, the corresponding results can be misleading and might present safety risks, especially regarding presurgical planning and evaluation. Several methods for specific signal processing have been proposed to tackle these problems, like Independent Component Analysis [93] for artifacts removal and Faraday/empty room recordings, in order to resolve background and instrument noise. However, unrelated signal noise from inhibitory brain functions can be still present, while critical signal properties could be embedded in the identified artifact-related independent components and thus be removed alongside them $[9,94]$. 
The number of electrodes and the number of dipoles defined by the source model creation is also crucial. As described in the previous sections, the number of source points vastly exceeds the number of electrodes and, thus, the solution is never unique and can be only estimated using priors (spatial, mathematical or anatomical). Furthermore, a very high number of source points would increase the resolution of the ESI solution, but would also increase the complexity of the process. More specifically, a small number of dipoles would result in lower spatial resolution and location accuracy, evidently making the produced solution more prone to misinterpretations, but require less memory and computational time to calculate. On the other hand, a higher number of source points leads to solutions with higher spatial resolution with smaller sparsity of the brain activity around each computed point, due to the larger number of neighboring points [9]. The main reason for the increased accuracy is that the Leadfield matrix is more complete and is calculated more thoroughly, indubitably requiring additional resources. As such, there is a fine balance on the computational cost and the ESI output where one has to consider minimizing the cortical activity sparsity (i.e., the extent of the source localization activation in terms of brain area surface) at the expense of high computational complexity. Leadfield calculation is also dependent on the number of electrode channels. The actual number of electrodes contributes significantly in the accuracy of the ESI, with limited channel number leading to the mislocalization of sources [40]. However, even if Low-Density ESI (LD-ESI) is less accurate compared to High-Density ESI (HD-ESI), LD-ESI is far more accessible and it could provide enough information given the appropriate recording time. Specifically, in hospital settings where High-Density EEG is unavailable, methods that could harness the power of a smaller number of electrodes could boost the usability of ESI. In Figure 2, different paradigms of electrode and source point numbers are displayed.

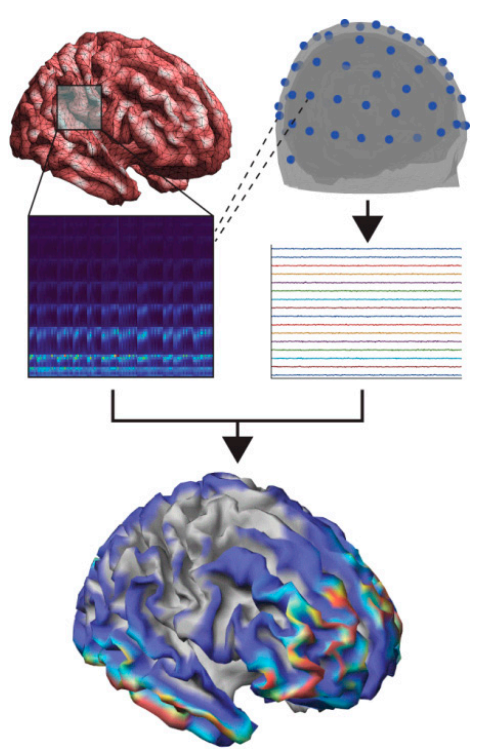

(a) Low-Density EEG with coarse resolution source model

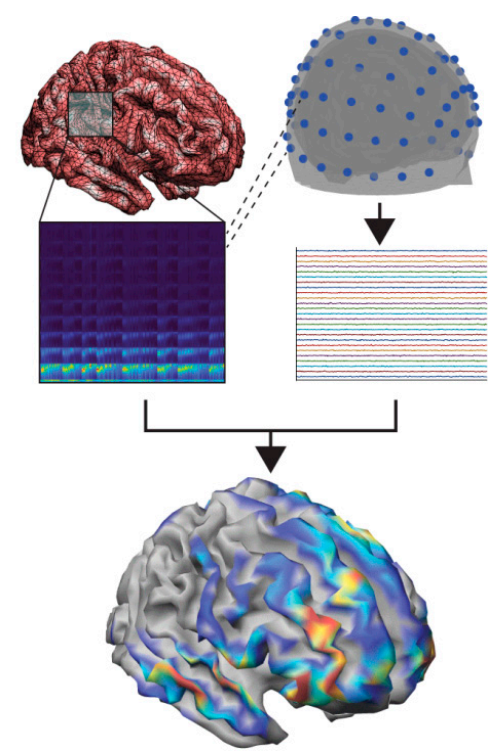

(b) Medium-Density EEG with normal resolution source model

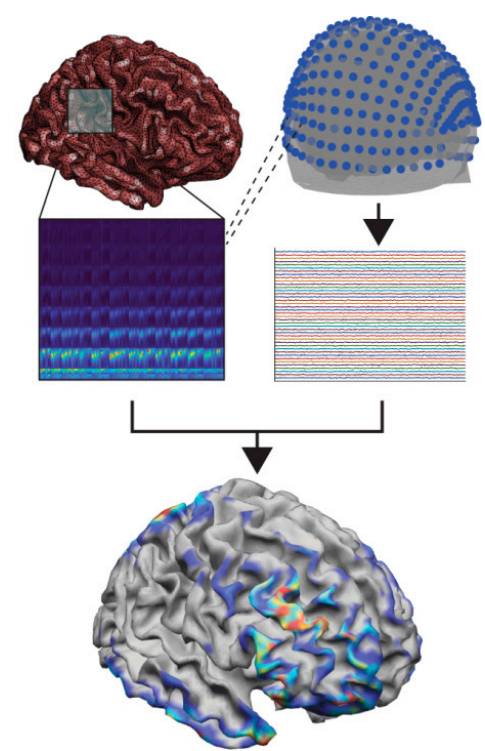

(c) High-Density EEG with fine resolution source model

Figure 2. Examples of how the number of electrodes and dipoles (source points) affect the ESI output. In each panel the calculated Leadfield matrix (heat map) depends on the electrode channels and the source points corresponding to a specific brain surface. In turn, the EEG recordings deriving from the different sensor settings are combined with the Leadfield matrix to estimate the locations of activity in the brain. In (a), a small number of electrode channels and source points leads to a more sparse and lower resolution outcome, while in (b), Medium-Density EEG and more dipoles refine the resulting solution and in (c), High-Density EEG and ultra-high resolution of the source model produces a solution with high spatial resolution and accuracy. 
Equally important is the co-registration of EEG sensors, i.e., the location of the electrodes, related to the head model and how coherent the electrode alignment is with the actual position of the electrodes during the EEG recordings. In this regard, minor misplacements during computational modeling could lead in inaccurate solutions [95]. New techniques and pipelines include 3D scanners in order to perform digitization of electrodes correctly and eliminate registration errors from the analysis $[96,97]$. Nevertheless, such systems are often very expensive, whereas the electrode alignment is done interactively [89]. It must also be taken into account that correct calculations of ESI relies on conductivity values that are provided to the head model [95]. On this premise, anatomical MRI could contribute to the correct solution of the forward (and as a result the inverse) problem, since the information concerning the thickness and conductivities of each head tissue is needed for the source localization computation [40]. For this reason, it is optimal to use subject-specific MRI in order to reconstruct the individual head model, additionally incorporating the anisotropic properties of the skull and white matter [36,98]. However, in several cases, individual MRI is unavailable or the head model cannot be created, due to brain abnormalities and impairments, such as brain malformation or tumors. In such conditions, pre-existing template solutions, such as standardized head models, could be used, albeit results could be controversial or designate non-existent areas [99].

Concerning the current state-of-the-arts methods presented in this paper, several limitations are still present. As far as the pattern recognition approaches are concerned, the main obstacle for effective localization is the lack of labeled real data to be used in order to train the Deep Learning architectures. Annotating real EEG data with the true source locations, for instance via simultaneous EEG-fMRI recordings, could be an encumbering task, but crucial in the progress of Deep Learning approaches in ESI. On the contrary, Bayesian Models provide enhanced localization accuracy, though these methods are extremely time-consuming in solving the inverse problem, which is an unbearable obstacle for real-time applications.

Recent advances focus towards this direction, increasing the efficiency of the algorithms employed. Future multi-modal approaches could combine the advantages of both data-driven and model-driven methods, exploiting the localization precision of statistical methods applied in Bayesian models with the computational speed of the Deep Learning processes.

\section{Conclusions}

In this paper, we present a review of recent approaches in solving both the forward and the inverse problem of EEG source imaging. Moreover, we tried to encapsulate the progress of ESI implementations in both clinical and cognitive research applications, while pointing out present challenges and limitations. As such, although state-of-the-art advancements demonstrate progress to a high degree in both the computational speed and precision aspects of ESI procedures, and further developments need to be incorporated into the source localization frameworks so that the new technologies can adapt to the existing protocols. Future mathematical approaches, computational methods and integration of source localization with other medical imaging methods, could enhance the value of ESI, resulting in significant progress in neuroscience, rehabilitation techniques and brain perception. By doing so, the high temporal resolution of a spatially accurate ESI would not only significantly improve cognitive evaluation and clinical applications, but also serve as a progressive step to explore the frontiers of the human mind.

Author Contributions: I.K. and G.K.M. had the original idea for the study. G.K.M. and E.M.V. defined the scope of the systematic review. I.Z. and E.M.V. performed the literature search and extraction. I.Z. and I.K. made the literature synthesis. I.Z. and G.K.M. drafted the paper, I.Z., I.K. and E.M.V. did the writing-review and editing. I.K. prepared the figures and G.K.M. supervised the manuscript preparation. All authors have read and agreed to the published version of the manuscript.

Funding: This research received no external funding. 
Institutional Review Board Statement: Not applicable.

Informed Consent Statement: Not applicable.

Data Availability Statement: Not applicable.

Conflicts of Interest: The authors declare no conflict of interest.

\section{References}

1. Mitzdorf, U. Current Source-Density Method and Application in Cat Cerebral Cortex: Investigation of Evoked Potentials and EEG Phenomena. Physiol. Rev. 1985, 65, 37-100. [CrossRef] [PubMed]

2. Helmholtz, H. Ueber Einige Gesetze Der Vertheilung Elektrischer Ströme in Körperlichen Leitern Mit Anwendung Auf Die Thierisch-Elektrischen Versuche. Ann. Der Phys. 1853, 165, 211-233. [CrossRef]

3. Hansen, S.T.; Hemakom, A.; Gylling Safeldt, M.; Krohne, L.K.; Madsen, K.H.; Siebner, H.R.; Mandic, D.P.; Hansen, L.K. Unmixing Oscillatory Brain Activity by EEG Source Localization and Empirical Mode Decomposition. Comput. Intell. Neurosci. 2019, 2019, 1-15. [CrossRef] [PubMed]

4. Mahjoory, K.; Nikulin, V.V.; Botrel, L.; Linkenkaer-Hansen, K.; Fato, M.M.; Haufe, S. Consistency of EEG Source Localization and Connectivity Estimates. NeuroImage 2017, 152, 590-601. [CrossRef]

5. Stropahl, M.; Bauer, A.-K.R.; Debener, S.; Bleichner, M.G. Source-Modeling Auditory Processes of EEG Data Using EEGLAB and Brainstorm. Front. Neurosci. 2018, 12. [CrossRef]

6. Michel, C.M.; He, B. EEG Mapping and Source Imaging. In Niedermeyer's Electroencephalography: Basic Principles, Clinical Applications, and Related Fields, 7th ed.; Schomer, D.L., Silva, F.H.L., Eds.; Oxford University Press: New York, NY, USA, 2018; ISBN 978-0-19-022848-4.

7. Nunez, P.L.; Srinivasan, R. Electric Fields of the Brain: The Neurophysics of EEG, 2nd ed.; Oxford University Press: New York, NY, USA, 2006; ISBN 978-0-19-505038-7.

8. Phillips, C.; Rugg, M.D.; Friston, K.J. Anatomically Informed Basis Functions for EEG Source Localization: Combining Functional and Anatomical Constraints. NeuroImage 2002, 16, 678-695. [CrossRef]

9. Michel, C.M.; Brunet, D. EEG Source Imaging: A Practical Review of the Analysis Steps. Front. Neurol. 2019, 10. [CrossRef]

10. Cui, S.; Duan, L.; Gong, B.; Qiao, Y.; Xu, F.; Chen, J.; Wang, C. EEG Source Localization Using Spatio-Temporal Neural Network. China Commun. 2019, 16, 131-143. [CrossRef]

11. Hecker, L.; Rupprecht, R.; Tebartz van Elst, L.; Kornmeier, J. ConvDip: A Convolutional Neural Network for Better M/EEG Source Imaging. bioRxiv 2020. [CrossRef]

12. Costa, F.; Batatia, H.; Oberlin, T.; D'Giano, C.; Tourneret, J.-Y. Bayesian EEG Source Localization Using a Structured Sparsity Prior. NeuroImage 2017, 144, 142-152. [CrossRef]

13. Kar, R.; Konar, A.; Chakraborty, A.; Bhattacharya, B.S.; Nagar, A.K. EEG Source Localization by Memory Network Analysis of Subjects Engaged in Perceiving Emotions from Facial Expressions. In Proceedings of the 2015 International Joint Conference on Neural Networks (IJCNN), Killarney, Ireland, 12-17 July 2015; pp. 1-8.

14. Ary, J.P.; Klein, S.A.; Fender, D.H. Location of Sources of Evoked Scalp Potentials: Corrections for Skull and Scalp Thicknesses. IEEE Trans. Biomed. Eng. 1981, BME-28, 447-452. [CrossRef] [PubMed]

15. Cho, J.-H.; Vorwerk, J.; Wolters, C.H.; Knösche, T.R. Influence of the Head Model on EEG and MEG Source Connectivity Analyses. NeuroImage 2015, 110, 60-77. [CrossRef] [PubMed]

16. Vorwerk, J.; Cho, J.-H.; Rampp, S.; Hamer, H.; Knösche, T.R.; Wolters, C.H. A Guideline for Head Volume Conductor Modeling in EEG and MEG. NeuroImage 2014, 100, 590-607. [CrossRef]

17. Hamalainen, M.S.; Sarvas, J. Realistic Conductivity Geometry Model of the Human Head for Interpretation of Neuromagnetic Data. IEEE Trans. Biomed. Eng. 1989, 36, 165-171. [CrossRef] [PubMed]

18. Saleheen, H.I.; Ng, K.T. New Finite Difference Formulations for General Inhomogeneous Anisotropic Bioelectric Problems. IEEE Trans. Biomed. Eng. 1997, 44, 800-809. [CrossRef]

19. Fuchs, M.; Wagner, M.; Kastner, J. Boundary Element Method Volume Conductor Models for EEG Source Reconstruction. Clin. Neurophysiol. 2001, 112, 1400-1407. [CrossRef]

20. Rullmann, M.; Anwander, A.; Dannhauer, M.; Warfield, S.K.; Duffy, F.H.; Wolters, C.H. EEG Source Analysis of Epileptiform Activity Using a $1 \mathrm{Mm}$ Anisotropic Hexahedra Finite Element Head Model. NeuroImage 2009, 44, 399-410. [CrossRef]

21. Wong, H.-S.; Ma, B.; Yu, Z.; Yeung, P.F.; Ip, H.H.S. 3-D Head Model Retrieval Using a Single Face View Query. IEEE Trans. Multimed. 2007, 9, 1026-1036. [CrossRef]

22. Nara, T.; Ando, S. A Projective Method for an Inverse Source Problem of the Poisson Equation. Inverse Probl. 2003, 19, 355-369. [CrossRef]

23. Saase, V.; Wenz, H.; Ganslandt, T.; Groden, C.; Maros, M.E. Simple Statistical Methods for Unsupervised Brain Anomaly Detection on MRI Are Competitive to Deep Learning Methods. arXiv 2020, arXiv:2011.12735.

24. Vorwerk, J.; Oostenveld, R.; Piastra, M.C.; Magyari, L.; Wolters, C.H. The FieldTrip-SimBio Pipeline for EEG Forward Solutions. BioMedical Eng. OnLine 2018, 17, 37. [CrossRef] [PubMed]

25. Huang, Y.; Parra, L.C.; Haufe, S. The New York Head-A Precise Standardized Volume Conductor Model for EEG Source Localization and TES Targeting. NeuroImage 2016, 140, 150-162. [CrossRef] [PubMed] 
26. Beltrachini, L. A Finite Element Solution of the Forward Problem in EEG for Multipolar Sources. IEEE Trans. Neural Syst. Rehabil. Eng. 2019, 27, 368-377. [CrossRef] [PubMed]

27. Birot, G.; Spinelli, L.; Vulliémoz, S.; Mégevand, P.; Brunet, D.; Seeck, M.; Michel, C.M. Head Model and Electrical Source Imaging: A Study of 38 Epileptic Patients. NeuroImage Clin. 2014, 5, 77-83. [CrossRef] [PubMed]

28. Engwer, C.; Vorwerk, J.; Ludewig, J.; Wolters, C.H. A Discontinuous Galerkin Method to Solve the EEG Forward Problem Using the Subtraction Approach. SIAM J. Sci. Comput. 2017, 39, B138-B164. [CrossRef]

29. Vorwerk, J.; Engwer, C.; Pursiainen, S.; Wolters, C.H. A Mixed Finite Element Method to Solve the EEG Forward Problem. IEEE Trans. Med Imaging 2017, 36, 930-941. [CrossRef]

30. Beltrachini, L. The Analytical Subtraction Approach for Solving the Forward Problem in EEG. J. Neural Eng. 2019, 16, 056029. [CrossRef]

31. Miinalainen, T.; Rezaei, A.; Us, D.; Nüßing, A.; Engwer, C.; Wolters, C.H.; Pursiainen, S. A Realistic, Accurate and Fast Source Modeling Approach for the EEG Forward Problem. NeuroImage 2019, 184, 56-67. [CrossRef]

32. Vanrumste, B.; Van Hoey, G.; Van de Walle, R.; D'Havè, M.R.P.; Lemahieu, I.A.; Boon, P.A.J.M. The Validation of the Finite Difference Method and Reciprocity for Solving the Inverse Problem in EEG Dipole Source Analysis. Brain Topogr. 2001, 14, 83-92. [CrossRef]

33. Cuartas Morales, E.; Acosta-Medina, C.D.; Castellanos-Dominguez, G.; Mantini, D. A Finite-Difference Solution for the EEG Forward Problem in Inhomogeneous Anisotropic Media. Brain Topogr. 2019, 32, 229-239. [CrossRef]

34. Pillain, A.; Rahmouni, L.; Andriulli, F. Handling Anisotropic Conductivities in the EEG Forward Problem with a Symmetric Formulation. Phys. Med. Biol. 2019, 64, 035022. [CrossRef] [PubMed]

35. Montes-Restrepo, V.; van Mierlo, P.; Strobbe, G.; Staelens, S.; Vandenberghe, S.; Hallez, H. Influence of Skull Modeling Approaches on EEG Source Localization. Brain Topogr. 2014, 27, 95-111. [CrossRef]

36. Rimpiläinen, V.; Koulouri, A.; Lucka, F.; Kaipio, J.P.; Wolters, C.H. Improved EEG Source Localization with Bayesian Uncertainty Modelling of Unknown Skull Conductivity. NeuroImage 2019, 188, 252-260. [CrossRef]

37. Fender, D.H. Source Localization of Brain Electrical Activity. In Handbook of Electroencephalography and Clinical Neurophysiology; Elsevier: Amsterdam, The Netherlands, 1987; pp. 355-403.

38. Grech, R.; Cassar, T.; Muscat, J.; Camilleri, K.P.; Fabri, S.G.; Zervakis, M.; Xanthopoulos, P.; Sakkalis, V.; Vanrumste, B. Review on Solving the Inverse Problem in EEG Source Analysis. J. NeuroEngineering Rehabil. 2008, 5, 25. [CrossRef]

39. Michel, C.M.; Murray, M.M.; Lantz, G.; Gonzalez, S.; Spinelli, L.; Grave de Peralta, R. EEG Source Imaging. Clin. Neurophysiol. 2004, 115, 2195-2222. [CrossRef]

40. Hämäläinen, M.S.; Ilmoniemi, R.J. Interpreting Magnetic Fields of the Brain: Minimum Norm Estimates. Med. Biol. Eng. Comput. 1994, 32, 35-42. [CrossRef]

41. Fuchs, M.; Wagner, M.; Köhler, T.; Wischmann, H.-A. Linear and Nonlinear Current Density Reconstructions. J. Clin. Neurophysiol. 1999, 16, 267-295. [CrossRef]

42. Lin, F.-H.; Witzel, T.; Ahlfors, S.P.; Stufflebeam, S.M.; Belliveau, J.W.; Hämäläinen, M.S. Assessing and Improving the Spatial Accuracy in MEG Source Localization by Depth-Weighted Minimum-Norm Estimates. NeuroImage 2006, 31, 160-171. [CrossRef]

43. Pascual-Marqui, R.D.; Michel, C.M.; Lehmann, D. Low Resolution Electromagnetic Tomography: A New Method for Localizing Electrical Activity in the Brain. Int. J. Psychophysiol. 1994, 18, 49-65. [CrossRef]

44. Pascual-Marqui, R.D.; Lehmann, D.; Koukkou, M.; Kochi, K.; Anderer, P.; Saletu, B.; Tanaka, H.; Hirata, K.; John, E.R.; Prichep, L.; et al. Assessing Interactions in the Brain with Exact Low-Resolution Electromagnetic Tomography. Philos. Trans. R. Soc. A Math. Phys. Eng. Sci. 2011, 369, 3768-3784. [CrossRef]

45. Vrba, J.; Robinson, S.E. Signal Processing in Magnetoencephalography. Methods 2001, 25, 249-271. [CrossRef]

46. Huang, G.; Yu, Z.L.; Wu, W.; Liu, K.; Gu, Z.; Qi, F.; Li, Y.; Liang, J. Electromagnetic Source Imaging via a Data-Synthesis-Based Denoising Autoencoder. arXiv 2021, arXiv:2010.12876.

47. Wipf, D.P.; Owen, J.P.; Attias, H.T.; Sekihara, K.; Nagarajan, S.S. Robust Bayesian Estimation of the Location, Orientation, and Time Course of Multiple Correlated Neural Sources Using MEG. NeuroImage 2010, 49, 641-655. [CrossRef]

48. Pirondini, E.; Babadi, B.; Obregon-Henao, G.; Lamus, C.; Malik, W.Q.; Hämäläinen, M.S.; Purdon, P.L. Computationally Efficient Algorithms for Sparse, Dynamic Solutions to the EEG Source Localization Problem. IEEE Trans. Biomed. Eng. 2018, 65, 1359-1372. [CrossRef]

49. Ebrahimzadeh, E.; Shams, M.; Rahimpour Jounghani, A.; Fayaz, F.; Mirbagheri, M.; Hakimi, N.; Hashemi Fesharaki, S.S.; Soltanian-Zadeh, H. Epilepsy Presurgical Evaluation of Patients with Complex Source Localization by a Novel Component-Based EEG-FMRI Approach. Iran J Radiol 2019, 16. [CrossRef]

50. Plummer, C.; Vogrin, S.J.; Woods, W.P.; Murphy, M.A.; Cook, M.J.; Liley, D.T.J. Interictal and Ictal Source Localization for Epilepsy Surgery Using High-Density EEG with MEG: A Prospective Long-Term Study. Brain 2019, 142, 932-951. [CrossRef]

51. Nemtsas, P.; Birot, G.; Pittau, F.; Michel, C.M.; Schaller, K.; Vulliemoz, S.; Kimiskidis, V.K.; Seeck, M. Source Localization of Ictal Epileptic Activity Based on High-Density Scalp EEG Data. Epilepsia 2017, 58, 1027-1036. [CrossRef]

52. Centeno, M.; Tierney, T.M.; Perani, S.; Shamshiri, E.A.; Pier, K.S.; Wilkinson, C.; Konn, D.; Vulliemoz, S.; Grouiller, F.; Lemieux, L.; et al. Combined Electroencephalography-Functional Magnetic Resonance Imaging and Electrical Source Imaging Improves Localization of Pediatric Focal Epilepsy. Ann. Neurol. 2017, 82, 278-287. [CrossRef]

53. Michel, C.M.; He, B. EEG Mapping and Source Imaging; Oxford University Press: New York, NY, USA, 2017; ISBN 978-0-19-022851-4. 
54. Foged, M.T.; Martens, T.; Pinborg, L.H.; Hamrouni, N.; Litman, M.; Rubboli, G.; Leffers, A.-M.; Ryvlin, P.; Jespersen, B.; Paulson, O.B.; et al. Diagnostic Added Value of Electrical Source Imaging in Presurgical Evaluation of Patients with Epilepsy: A Prospective Study. Clin. Neurophysiol. 2020, 131, 324-329. [CrossRef]

55. Mégevand, P.; Seeck, M. Electric Source Imaging for Presurgical Epilepsy Evaluation: Current Status and Future Prospects. Expert Rev. Med Devices 2020, 17, 405-412. [CrossRef]

56. Jun, Y.-H.; Eom, T.-H.; Kim, Y.-H.; Chung, S.-Y.; Lee, I.-G.; Kim, J.-M. Source Localization of Epileptiform Discharges in Childhood Absence Epilepsy Using a Distributed Source Model: A Standardized, Low-Resolution, Brain Electromagnetic Tomography (SLORETA) Study. Neurol Sci 2019, 40, 993-1000. [CrossRef] [PubMed]

57. van Mierlo, P.; Vorderwülbecke, B.J.; Staljanssens, W.; Seeck, M.; Vulliémoz, S. Ictal EEG Source Localization in Focal Epilepsy: Review and Future Perspectives. Clin. Neurophysiol. 2020, 131, 2600-2616. [CrossRef] [PubMed]

58. Coito, A.; Biethahn, S.; Tepperberg, J.; Carboni, M.; Roelcke, U.; Seeck, M.; van Mierlo, P.; Gschwind, M.; Vulliemoz, S. Interictal Epileptogenic Zone Localization in Patients with Focal Epilepsy Using Electric Source Imaging and Directed Functional Connectivity from Low-Density EEG. Epilepsia Open 2019, 4, 281-292. [CrossRef] [PubMed]

59. Staljanssens, W.; Strobbe, G.; Van Holen, R.; Keereman, V.; Gadeyne, S.; Carrette, E.; Meurs, A.; Pittau, F.; Momjian, S.; Seeck, M.; et al. EEG Source Connectivity to Localize the Seizure Onset Zone in Patients with Drug Resistant Epilepsy. NeuroImage Clin. 2017, 16, 689-698. [CrossRef]

60. Vespa, S.; Baroumand, A.G.; Ferrao Santos, S.; Vrielynck, P.; de Tourtchaninoff, M.; Feys, O.; Strobbe, G.; Raftopoulos, C.; van Mierlo, P.; El Tahry, R. Ictal EEG Source Imaging and Connectivity to Localize the Seizure Onset Zone in Extratemporal Lobe Epilepsy. Seizure 2020, 78, 18-30. [CrossRef]

61. Galaris, E.; Gallos, I.; Myatchin, I.; Lagae, L.; Siettos, C. EEG Source Localization Analysis in Epileptic Children during a Visual Working-Memory Task. arXiv 2020, arXiv:2005.11186.

62. Zhou, J.; Liu, S.; Ng, K.K.; Wang, J. Applications of Resting-State Functional Connectivity to Neurodegenerative Disease. Neuroimaging Clin. 2017, 27, 663-683. [CrossRef]

63. Tsolaki, A.C.; Kosmidou, V.; Kompatsiaris, I.Y.; Papadaniil, C.; Hadjileontiadis, L.; Adam, A.; Tsolaki, M. Brain Source Localization of MMN and P300 ERPs in Mild Cognitive Impairment and Alzheimer's Disease: A High-Density EEG Approach. Neurobiol Aging 2017, 55, 190-201. [CrossRef]

64. Hata, M.; Kazui, H.; Tanaka, T.; Ishii, R.; Canuet, L.; Pascual-Marqui, R.D.; Aoki, Y.; Ikeda, S.; Kanemoto, H.; Yoshiyama, K.; et al. Functional Connectivity Assessed by Resting State EEG Correlates with Cognitive Decline of Alzheimer's Disease-An ELORETA Study. Clin. Neurophysiol. 2016, 127, 1269-1278. [CrossRef]

65. La Foresta, F.; Morabito, F.C.; Marino, S.; Dattola, S. High-Density EEG Signal Processing Based on Active-Source Reconstruction for Brain Network Analysis in Alzheimer's Disease. Electronics 2019, 8, 1031. [CrossRef]

66. Kang, X.; Boly, M.; Findlay, G.; Jones, B.; Gjini, K.; Maganti, R.; Struck, A.F. Quantitative Spatio-Temporal Characterization of Epileptic Spikes Using High Density EEG: Differences between NREM Sleep and REM Sleep. Sci. Rep. 2020, 10, 1673. [CrossRef] [PubMed]

67. Fernandez Guerrero, A.; Achermann, P. Brain Dynamics during the Sleep Onset Transition: An EEG Source Localization Study. Neurobiol. Sleep Circadian Rhythm. 2019, 6, 24-34. [CrossRef]

68. Bersagliere, A.; Pascual-Marqui, R.D.; Tarokh, L.; Achermann, P. Mapping Slow Waves by EEG Topography and Source Localization: Effects of Sleep Deprivation. Brain Topogr 2018, 31, 257-269. [CrossRef]

69. Brancaccio, A.; Tabarelli, D.; Bigica, M.; Baldauf, D. Cortical Source Localization of Sleep-Stage Specific Oscillatory Activity. Sci. Rep. 2020, 10, 6976. [CrossRef]

70. Klinzing, J.G.; Mölle, M.; Weber, F.; Supp, G.; Hipp, J.F.; Engel, A.K.; Born, J. Spindle Activity Phase-Locked to Sleep Slow Oscillations. NeuroImage 2016, 134, 607-616. [CrossRef] [PubMed]

71. Murphy, M.; Riedner, B.A.; Huber, R.; Massimini, M.; Ferrarelli, F.; Tononi, G. Source Modeling Sleep Slow Waves. Proc. Natl. Acad. Sci. USA 2009, 106, 1608-1613. [CrossRef]

72. Castelnovo, A.; Riedner, B.A.; Smith, R.F.; Tononi, G.; Boly, M.; Benca, R.M. Scalp and Source Power Topography in Sleepwalking and Sleep Terrors: A High-Density EEG Study. Sleep 2016, 39, 1815-1825. [CrossRef]

73. Handiru, V.S.; Vinod, A.P.; Guan, C. Multi-Direction Hand Movement Classification Using EEG-Based Source Space Analysis. In Proceedings of the 2016 38th Annual International Conference of the IEEE Engineering in Medicine and Biology Society (EMBC), Orlando, FL, USA, 16-20 August 2016; pp. 4551-4554.

74. Li, M.-A.; Wang, Y.-F.; Jia, S.-M.; Sun, Y.-J.; Yang, J.-F. Decoding of Motor Imagery EEG Based on Brain Source Estimation. Neurocomputing 2019, 339, 182-193. [CrossRef]

75. Edelman, B.J.; Baxter, B.; He, B. EEG Source Imaging Enhances the Decoding of Complex Right-Hand Motor Imagery Tasks. IEEE Trans. Biomed. Eng. 2016, 63, 4-14. [CrossRef] [PubMed]

76. Hou, Y.; Zhou, L.; Jia, S.; Lun, X. A Novel Approach of Decoding EEG Four-Class Motor Imagery Tasks via Scout ESI and CNN. J. Neural Eng. 2020, 17, 016048. [CrossRef]

77. Antelis, J.M.; Montesano, L.; Ramos-Murguialday, A.; Birbaumer, N.; Minguez, J. Decoding Upper Limb Movement Attempt From EEG Measurements of the Contralesional Motor Cortex in Chronic Stroke Patients. IEEE Trans. Biomed. Eng. 2017, 64, 99-111. [CrossRef] [PubMed] 
78. Mottaz, A.; Solcà, M.; Magnin, C.; Corbet, T.; Schnider, A.; Guggisberg, A.G. Neurofeedback Training of Alpha-Band Coherence Enhances Motor Performance. Clin. Neurophysiol. 2015, 126, 1754-1760. [CrossRef]

79. Dubovik, S.; Ptak, R.; Aboulafia, T.; Magnin, C.; Gillabert, N.; Allet, L.; Pignat, J.-M.; Schnider, A.; Guggisberg, A.G. EEG Alpha Band Synchrony Predicts Cognitive and Motor Performance in Patients with Ischemic Stroke. Behav Neurol 2013, 26, 187-189. [CrossRef]

80. Nicolo, P.; Rizk, S.; Magnin, C.; Pietro, M.D.; Schnider, A.; Guggisberg, A.G. Coherent Neural Oscillations Predict Future Motor and Language Improvement after Stroke. Brain 2015, 138, 3048-3060. [CrossRef]

81. Friedrich, J.; Beste, C. Paradoxical, Causal Effects of Sensory Gain Modulation on Motor Inhibitory Control-A TDCS, EEG-Source Localization Study. Sci. Rep. 2018, 8, 17486. [CrossRef] [PubMed]

82. Hong, X.; Wang, Y.; Sun, J.; Li, C.; Tong, S. Segregating Top-Down Selective Attention from Response Inhibition in a Spatial Cueing Go/NoGo Task: An ERP and Source Localization Study. Sci. Rep. 2017, 7, 9662. [CrossRef] [PubMed]

83. Sabeti, M.; Katebi, S.D.; Rastgar, K.; Azimifar, Z. A Multi-Resolution Approach to Localize Neural Sources of P300 Event-Related Brain Potential. Comput. Methods Programs Biomed. 2016, 133, 155-168. [CrossRef] [PubMed]

84. Herrmann, M.J.; Ehlis, A.-C.; Muehlberger, A.; Fallgatter, A.J. Source Localization of Early Stages of Face Processing. Brain Topogr 2005, 18, 77-85. [CrossRef]

85. Andersen, R.S.; Eliasen, A.U.; Pedersen, N.; Andersen, M.R.; Hansen, S.T.; Hansen, L.K. EEG Source Imaging Assists Decoding in a Face Recognition Task. In Proceedings of the 2017 IEEE International Conference on Acoustics, Speech and Signal Processing (ICASSP), New Orleans, LA, USA, 5-9 March 2017; pp. 939-943.

86. Calbi, M.; Siri, F.; Heimann, K.; Barratt, D.; Gallese, V.; Kolesnikov, A.; Umiltà, M.A. How Context Influences the Interpretation of Facial Expressions: A Source Localization High-Density EEG Study on the "Kuleshov Effect". Sci. Rep. 2019, 9, 2107. [CrossRef]

87. Monteiro, R.; Simões, M.; Andrade, J.; Castelo Branco, M. Processing of Facial Expressions in Autism: A Systematic Review of EEG/ERP Evidence. Rev J Autism Dev Disord 2017, 4, 255-276. [CrossRef]

88. Chikara, R.K.; Ko, L.-W. Modulation of the Visual to Auditory Human Inhibitory Brain Network: An EEG Dipole Source Localization Study. Brain Sci. 2019, 9, 216. [CrossRef]

89. Fahimi Hnazaee, M.; Khachatryan, E.; Van Hulle, M.M. Semantic Features Reveal Different Networks During Word Processing: An EEG Source Localization Study. Front. Hum. Neurosci. 2018, 12. [CrossRef]

90. Dimitrakopoulos, G.N.; Kakkos, I.; Dai, Z.; Lim, J.; deSouza, J.J.; Bezerianos, A.; Sun, Y. Task-Independent Mental Workload Classification Based Upon Common Multiband EEG Cortical Connectivity. IEEE Trans. Neural Syst. Rehabil. Eng. 2017, 25, 1940-1949. [CrossRef] [PubMed]

91. Kakkos, I.; Dimitrakopoulos, G.N.; Gao, L.; Zhang, Y.; Qi, P.; Matsopoulos, G.K.; Thakor, N.; Bezerianos, A.; Sun, Y. Mental Workload Drives Different Reorganizations of Functional Cortical Connectivity Between 2D and 3D Simulated Flight Experiments. IEEE Trans. Neural Syst. Rehabil. Eng. 2019, 27, 1704-1713. [CrossRef] [PubMed]

92. Whittingstall, K.; Stroink, G.; Gates, L.; Connolly, J.; Finley, A. Effects of Dipole Position, Orientation and Noise on the Accuracy of EEG Source Localization. BioMed. Eng. OnLine 2003, 2, 14. [CrossRef] [PubMed]

93. Delorme, A.; Sejnowski, T.; Makeig, S. Enhanced Detection of Artifacts in EEG Data Using Higher-Order Statistics and Independent Component Analysis. NeuroImage 2007, 34, 1443-1449. [CrossRef] [PubMed]

94. Jiang, X.; Bian, G.-B.; Tian, Z. Removal of Artifacts from EEG Signals: A Review. Sensors 2019, 19, 987. [CrossRef] [PubMed]

95. Akalin Acar, Z.; Makeig, S. Effects of Forward Model Errors on EEG Source Localization. Brain Topogr 2013, 26, 378-396. [CrossRef]

96. Homölle, S.; Oostenveld, R. Using a Structured-Light 3D Scanner to Improve EEG Source Modeling with More Accurate Electrode Positions. J. Neurosci. Methods 2019, 326, 108378. [CrossRef]

97. Cline, C.C.; Coogan, C.; He, B. EEG Electrode Digitization with Commercial Virtual Reality Hardware. PLoS ONE 2018, 13, e0207516. [CrossRef] [PubMed]

98. Lee, W.H.; Liu, Z.; Mueller, B.A.; Lim, K.; He, B. Influence of White Matter Anisotropic Conductivity on EEG Source Localization: Comparison to FMRI in Human Primary Visual Cortex. Clin. Neurophysiol. 2009, 120, 2071-2081. [CrossRef] [PubMed]

99. Brodbeck, V.; Spinelli, L.; Lascano, A.M.; Wissmeier, M.; Vargas, M.-I.; Vulliemoz, S.; Pollo, C.; Schaller, K.; Michel, C.M.; Seeck, M. Electroencephalographic Source Imaging: A Prospective Study of 152 Operated Epileptic Patients. Brain 2011, 134, $2887-2897$. [CrossRef] [PubMed] 